\title{
New Pre-injector of the KEK 2.5-GeV Linac and its Performance
}

\author{
S. Ohsawa, I. Abe, S. Anami, J.-Y. Choi, A. Enomoto, K. Furukawa, \\ H. Hanaki, K. Kakihara, N. Kamikubota, T. Kamitani, H. Kobayashi, \\ Y. Ogawa, T. Oogoe, I. Sato, T. Suwada, Y. Yamazaki, M. Yokota, \\ KEK, National Laboratory for High Energy Physics \\ Oho, 1-1 Tsukuba-shi, Ibaraki-ken, 305 Japan \\ and A. Asami \\ Naruto University of Education \\ Takashima, Naruto-cho, Naruto-shi, Tokushima-ken, 722 Japan
}

\section{Abstract}

The injection system (pre-injector) of the KEK 2.5-GeV linac has been upgraded so that we can investigate intense beam acceleration for the $\mathrm{KEK}$ B-factory project. It requires intense beams to achieve a short injection time in practice. An outline of the new pre-injector and its performance is given.

\section{INTRODUCTION}

The pre-injector of the $2.5-\mathrm{GeV}$ linac was extensively upgraded during the summer of 1992 [1]. We installed the new pre-injector during the summer maintenance period from July 11 to September 27, 1992. At first we completely removed almost all of the previous system in the tunnel. Entire devises, including a supporting girder, were replaced with new ones, except for 2 quadrupole magnets and 2 accelerating guides. The first test operation with beams was performed on September 24 to 25 , as scheduled. Everything was completed within the period, except for some small items. Normal operation started well on September 28 , as prearranged.

This is the first step toward upgrading the linac to meet the requirements of the KEK B-factory [2], which is presently under consideration as a future project. It requires intense beams, especially an intense $e^{-}$beam for producing positrons. It is, therefore, important to understand how highintensity $\mathrm{e}^{-}$beams can be accelerated in the linac, as well as any instabilities of the beams.

\section{NEW SYSTEM CONFIGURATION}

The layout of the new pre-injector is shown in Fig. 1. Improved items extended over a wide area [3]. The main items are described in the following.

\section{A. Electron Gun}

We changed the electron gun to a newly designed one, which is under operation at $190 \mathrm{kV}$. A ceramic insulator was changed from one $130 \mathrm{~mm}$ long with ICF-203 flanges to at one of $300 \mathrm{~mm}$ long with ICF-253 flanges. The cathode is a commercially available one (EIMAC Y-796).

\section{B. Bunching System}

We adopted a multiple bunching system that comprises double prebunchers and a high field buncher [1]. Our new system has several features: (1) The distance between the 2 nd prebuncher and the buncher was made as short as possible in order to obtain short bunches. This is also important to minimize any effect resulting from beam loading in the gun. If the beam energy is gradually reduced at the gun, multiple bunches are made at different phases. The shorter distance requires a stronger modulating field, which makes the loading effect relatively small. (2) The prebunchers were designed to have very low shunt impedances and large group velocities. The aim is to reduce any wake-field effects caused by an intense beam. (3) The buncher comprises two sections, each of which has a high field $(15 \mathrm{MV} / \mathrm{m})$. The beginning part is the bunching section, which has 6 cavities, including an input coupler. Each cavity has a different phase velocity, varying gradually from $0.77 c$ to $0.99 c$. A normal section with a phase velocity of $c$ directly follows the bunching section. (4) All of the prebunchers and buncher are of the travelingwave type. (5) The field asymmetry in the coupler cavities was compensated for by offsetting the coupler cylinders instead of making a counter slot on the cylinder wall [4].

The field strengths and phase velocities of the bunching system were determined by simulations

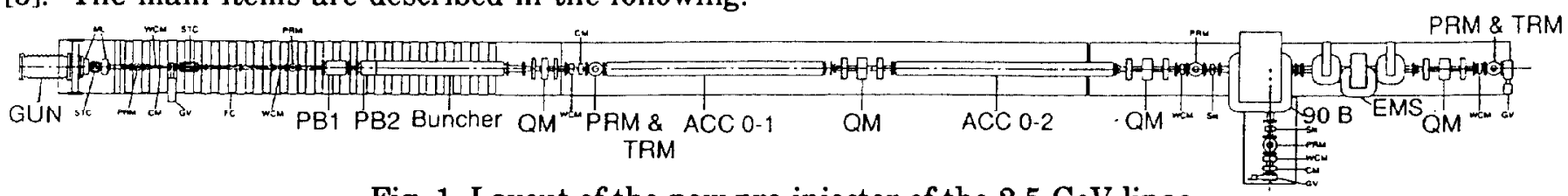

Fig. 1 Layout of the new pre-injector of the $2.5-\mathrm{GeV}$ linac 
with PARMELA. An example is shown in Figs. 2a and $2 \mathrm{~b}$. The simulation predicts a bunch length of $5^{\circ}$ (FWHM) at the buncher exit for a $200 \mathrm{keV} / 8 \mathrm{~A}$ beam from the gun [5]. The particles plotted in these figures reach just $90 \%$ of the initial particles, which indicates a high bunching efficiency.

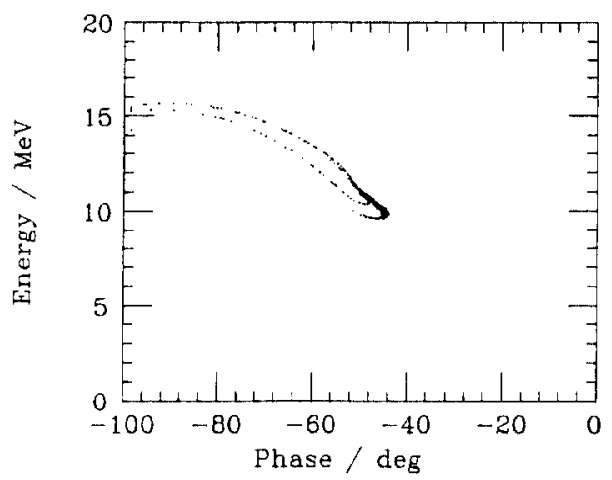

Fig. 2a Simulation example of energy distribution versus the buncher rf phase.

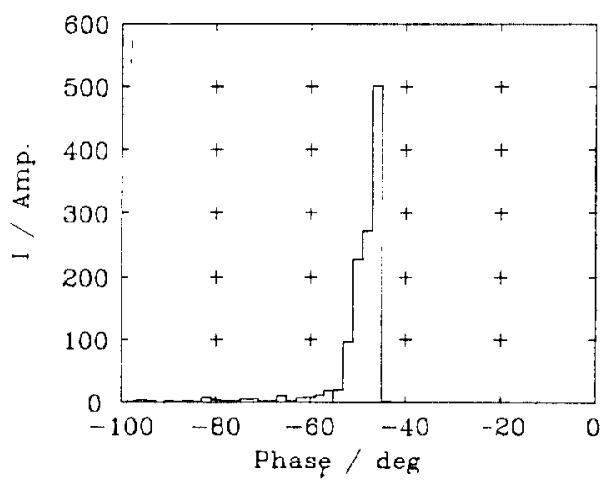

Fig. 2b Current density versus the rf phase in the same case. Each bin has a width of $2^{\circ}$.

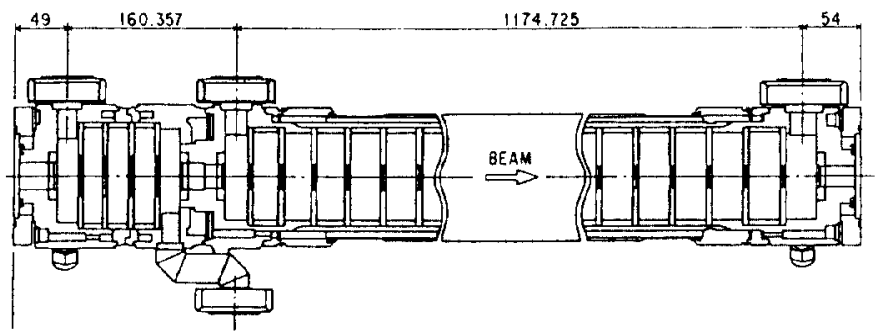

Fig. 3 Structure of the 2nd prebuncher and buncher, which are connected mechanically, but separated electrically.

\section{RF Adjusting System}

It is necessary to optimize the power levels and phases of microwaves fed into the prebunchers and the buncher. We employed equipment that is capable of changing the power level and the phase independently [3],[6]. This is useful for simplifying the rf optimization in the bunching section. Since a double prebuncher system was adopted, one more unit became additionally necessary for the rf adjusting system. This time we did not just add one unit for the system, but also rearranged the entire system.

The new $r f$ adjusting system is shown schematically in Fig. 4. It has the following additional features: (1) The power dividing ratio for the 2nd prebuncher and buncher, which is controlled by the first unit, can be changed continuously without any phase shift. (2) When the same unit is driven as a phase shifter, all of the rf phases in the bunching system are shifted simultaneously by the same amount. This property is very useful for phasing the beam with respect to the fixed rf phases in the following accelerating guides, since it does not affect the beam bunches.

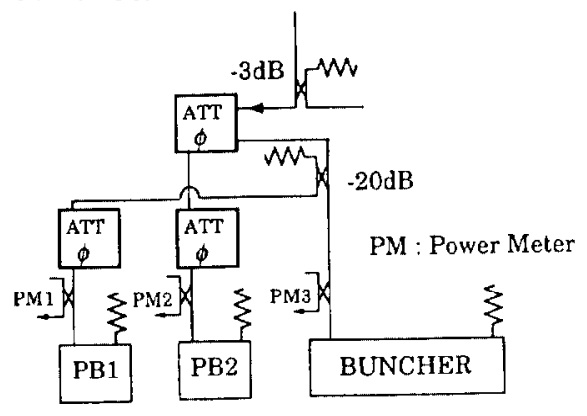

Fig. 4 Schematic diagram of the new rf adjusting system

\section{Beam Monitors}

As the beam intensity increases, it becomes more important to transport beams at the centers of the accelerating cavities in order to reduce some of the transverse wake-field effects. We added some fluorescent screen monitors, two of which have additionally polished stainless steel as the transition light emitter. A streak camera is used to analyze the transition light for measuring the bunch lengths [7].

\section{PERFORMANCE TEST}

\section{A. High-Current Acceleration}

After the pre-injector upgrade, high-current beam acceleration was performed with an 8-A/ 4-ns beam, which contained $9.3 \times 10^{10}$ electrons. The transport system was carefully adjusted only to the center of the 2nd sector, since the positronproduction target had been assumed to be installed somewhere up to that point. Electrons of $83 \%$ were accelerated to the 1st sector entrance and $73 \%$ to the 2nd sector entrance. The results are given in Fig. 5 . Monitor No. 11 is located at the 2nd sector center. After the upgrade, the beam currents increased as well as the transmission rate, as was expected. 
Up to the center of the 2 nd sector, beam profile deformation was not obvious on the screen monitors. In the following sectors, however, deformed profiles were observed, and the beam charge was decreased to $5.0 \mathrm{nC}$ at the center of the 4 th sector. The deformation that depended on the beam positions indicates that it was caused by wake fields.

Beams that contained much more charge were accelerated as a trial. They were longer beams with lower densities. As can be seen in Fig. 5, electrons of high rate ( 83 to $89 \%$ ) were accelerated to the preinjector exit. The beams were, however, considerably lost in the following sectors, especially in the first unit of the 1st sector. We are planing to install a quadrupole magnet there in order to improve the transmission rate. Beam loss is caused by a heavy beam-loading effect as well as transverse wake fields.

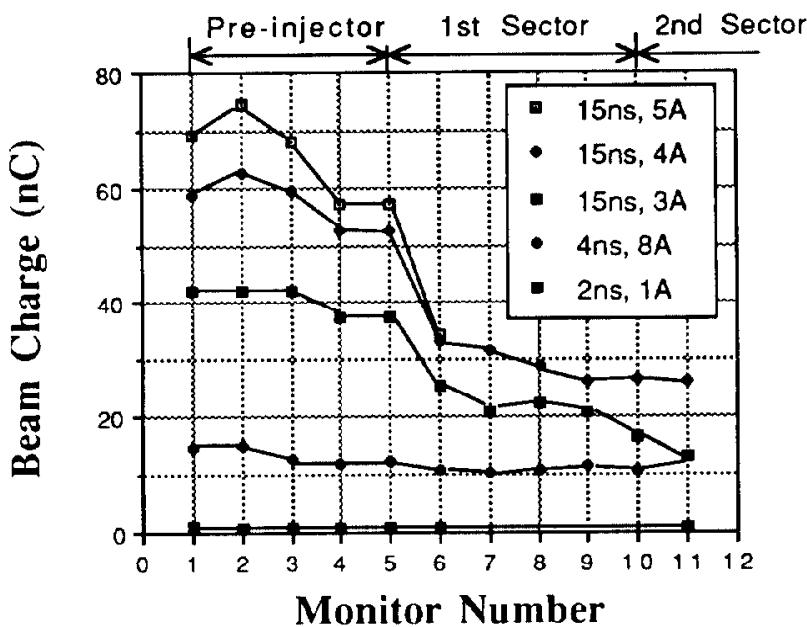

Fig. 5 High-charge beam acceleration after the preinjector upgrade.

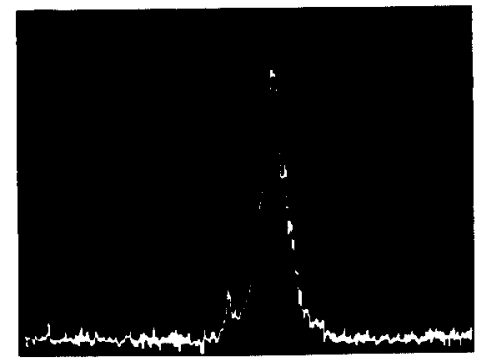

Fig. 6 One of the bunches of the 8-A/4-ns beam measured with a streak camera. It is a single-shot measurement.

\section{B. Bunch Length}

Beam bunch lengths were measured at the end of the pre-injector using the streak camera. Figure 6 shows one of the bunches in the 8-A/4-ns beam, which was taken, while analyzing the transition light. The measured bunch length (FWHM) is $9.8 \mathrm{ps}$, which is about twice as large as a prediction by a PARMELA simulation. The reason for the discrepancy is under investigation. Some reasons can be suggested: (1) incorrect bunching parameters, (2) wake-field effects, and (3) bunch-monitor performance.

\section{Emittance}

The beam emittance was measured just downstream from the buncher by the usual method with a screen monitor and a quadrupole magnet. The beam sizes were measured on the screen varying a focusing strength of the quadrupole magnet. Beam sizes were defined by the full width at half maximum in the beam-density distribution. For a 4-A/15-ns beam, the obtained emittances were $\varepsilon_{\mathrm{x}}=$ $45(\mathrm{~mm} \mathrm{mr})$ and $\varepsilon_{\mathrm{y}}=52(\mathrm{~mm} \mathrm{mr})$.

\section{FUTURE}

The KEK B-factory requires for injection an sband single-bunched $e^{-}$beam and an $e^{+}$beam of two or three bunches that are about $100 \mathrm{~ns}$ apart. Toward obtaining a single-bunched beam, we are presently preparing a $476-\mathrm{MHz} \mathrm{SHB}$ and its driver, which will be installed this summer. We will investigate soon what will occur in high-density single-bunched $\mathrm{e}^{-}$ beam acceleration at the present state of the $2.5-\mathrm{GeV}$ linac. The purpose is, of cause, to determine what should be improved so that our linac will meet its requirements.

\section{REFERENCES}

[1] S. Ohsawa et al., "Improvements to the Injection system of the KEK $2.5-\mathrm{GeV}$ Linac," Conference Record of the 16th International Linac Conference, 1992, pp. 91-93.

[2] A. Enomoto et al., "Linac Upgrade Plan for the KEK B-Factory," these proceedings.

[3] Photon Factory Activity Report 1992, KEK, to be published.

[4] H. Hanaki et al., "Computer Design of Coupler Cavities for a Traveling-Wave-Type Buncher," Conference Record of the 3rd European Particle Accelerator Conference, Vol. 2 , pp. 910-912.

[5] T. Kamitani et al., "A Simulation Study on Beam Bunching in the KEK 2.5-GeV Linac New Pre-injector," these proceedings.

[6] S. Ohsawa et al., "High-Power Hybrid Attenuator \& Phase-Shifter Systems," Conference Record of the 15th International Linac Conference, 1990, pp. 159-161.

[7] Y. Ogawa et al., "Beam Monitor Utilizing Transition Radiation," these proceedings. 